\title{
Synthesis of 2-phenylindoxyls
}

\author{
Michael C. Hewitt* and Liming Shao
}

Drug Discovery, Sepracor Inc., 84 Waterford Drive, Marlborough MA 01752, USA

E-mail: michael.hewitt@sepracor.com

\begin{abstract}
Described herein is a novel route to 2-phenylindoxyls. A previously undisclosed acid catalyzed reaction of $\mathrm{N}$-hydroxyalkyl-3-acetoxy-2-phenylindoles gave the desired 2-phenylindoxyls, the identity of which was confirmed by 2D-NMR. The product structure represents a scaffold that could be exploited in a pharmaceutical or academic setting.
\end{abstract}

Keywords: 2-phenylindoxyl, 2D-NMR, 2-phenylindole

\section{Introduction}

Indoles are perhaps the most widely distributed heterocycles in nature, as the amino acid tryptophan is a constituent of almost every protein. ${ }^{1}$ As such, the synthesis of indoles ${ }^{2}$ and indole analogs, such as indoxyl ${ }^{3}$ and oxindole, ${ }^{4}$ have received considerable attention from synthetic organic chemists.

A number of different routes to 2-phenylindoxyls have been reported, including the addition of phenyl Grignard reagents to isatin ${ }^{5}$ and alkyl grignard reagents to 2-phenyl-3H-indol3-ones. ${ }^{6}$ The 1,3-dipolar cycloaddition of 2-phenyl-3H-indol-3-one $\mathrm{N}$-oxides to electron deficient alkenes also yields 2-phenylindoxyl skeletons, albeit containing a cyclic N-O bond. ${ }^{7}$ Oxidative transformations yielding 2-phenylindoxyls include the reaction of 3-oxo-2,3dihydroindoles with aryllead triacetate, ${ }^{8}$ and the Baeyer-Villiger rearrangment of $\mathrm{N}$-acetyl-2phenyl-indole-3-carboxaldehyde. ${ }^{9} \quad N$-acetyl-2-phenylindoxyl can also be formed from the selective hydrolysis of 1,3-diacetylindole. ${ }^{10}$ The base-catalyzed rearrangement of $3 \mathrm{H}$-Indol-3-ols have also been reported - one of many possibilities available for the synthesis of 2,2'-diphenyl indoxyls. ${ }^{11}$

A relatively straightforward route to 2-phenylindoxyls would be a valuable addition to the repertoire of reactions available to synthetic chemists. In this vein, hydrolysis of a 3-acetoxy-2phenyl-indole precursor would seem to be a logical choice. It was previously reported by Sukari et al. that basic hydrolysis of 3-acetoxy-2-phenyl indoles 1 gave not the desired indoxyls, but rather dioxindoles 3 (Scheme 1). ${ }^{12}$ Initial hydrolysis of the 3-acetoxy group was followed by 
autooxidation and rearrangment. The autooxidation sequence $($ i.e. $1 \rightarrow 2$ ) had ample precedent in the transformation of indoxyl to the dye indigo. ${ }^{13}$

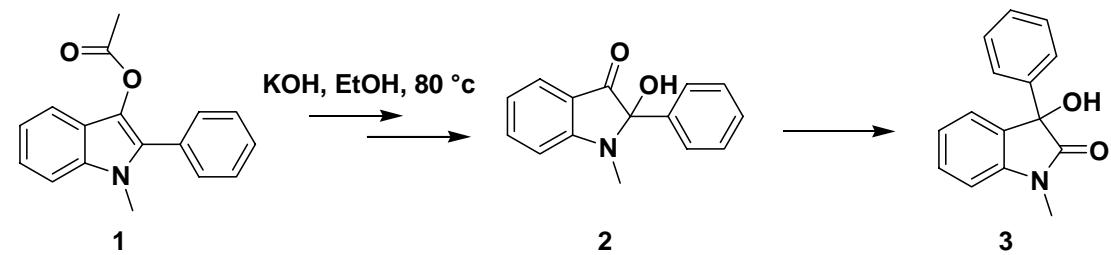

Scheme 1. Basic hydrolysis of 3-acetoxy indoles.

\section{Results and Discussion}

To the best of our knowledge the acidic hydrolysis of 3-acetyl-2-phenyl indoles had never been studied. Our investigation began with the synthesis of several $\mathrm{N}$-alkyl-2-phenyl indole derivatives (Scheme 2). The hydrolysis precursors were prepared in 2-3 steps from commercially available indoles via sequential $\mathrm{N}$-alkylation and oxidation with $\mathrm{Pb}(\mathrm{OAc})_{4}{ }^{12}$ Alkylation of 2phenyl indole $\mathbf{4}$ with cyclopropylmethyl bromide was followed by acetoxylation to give ester $\mathbf{5 a}$. Similarly, $N$-allylation, hydrogenation and acetoxylation provided ester $\mathbf{5 b}$.
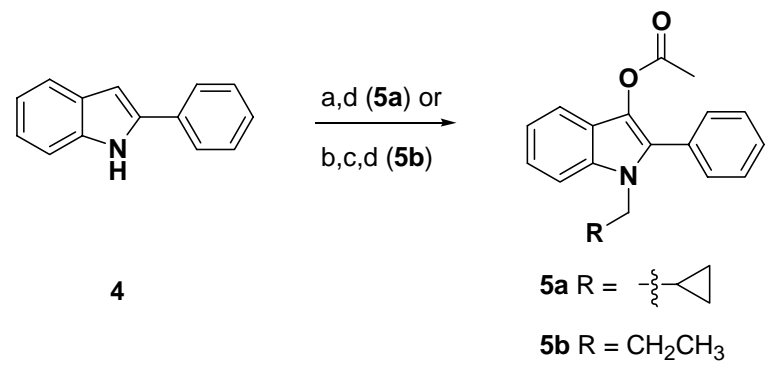

Scheme 2. Synthesis of $\mathrm{N}$-alkyl analogs. a) NaH, DMF, cyclopropylmethyl bromide. b) $\mathrm{NaH}$, DMF, allyl bromide. c) $\mathrm{H}_{2}, \mathrm{Pd} / \mathrm{C}$. d) $\mathrm{CH}_{2} \mathrm{Cl}_{2}, \mathrm{~Pb}(\mathrm{OAc})_{4}$.

Basic hydrolysis $\left(\mathrm{KOH}, \mathrm{EtOH}, 80{ }^{\circ} \mathrm{C}\right)$ of $\mathrm{N}$-propyl indole 5a gave a dioxindole, ${ }^{14}$ in line with the findings of Sukari (vida supra). Acidic hydrolysis of $N$-alkyl-3-acetyl substrates $\mathbf{5}$ were carried out in methanol with a $2 \mathrm{M}$ solution of $\mathrm{HCl}$ and provided a mixture of 2-phenylindoxyls 6 and 7. NMR $\left({ }^{1} \mathrm{H},{ }^{13} \mathrm{C}\right.$ and DEPT $)$ and GC-MS confirmed that the major product $6 \mathbf{b}$ possessed a methoxy group at the 2-position and the minor product $\mathbf{7 b}$ a hydroxyl group, respectively (Scheme 3). The structures of $\mathbf{6 a}$ and 7a were determined based on HPLC and GC-MS analysis and by analogy to the results seen for $\mathbf{6 b}$ and $\mathbf{7 b}$. To account for the formation of structures $\mathbf{6}$ and 7 , we theorized that a radical or cation was forming at the benzylic position and was reacting 
with solvent molecules. This prompted us to investigate whether the intermediate species could be trapped intramolecularly via attack by an alcohol tethered to the indole nitrogen.

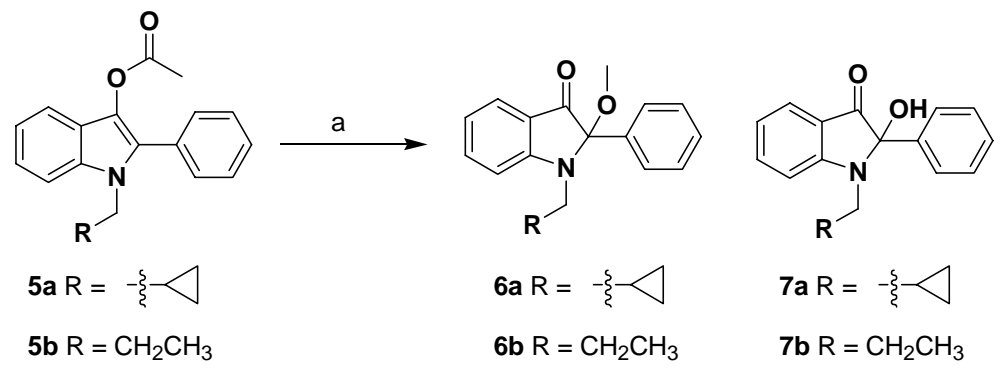

Scheme 3. Formation of 2-phenylindoxyls. a) 2M HCl, MeOH, RT.

Thus, we began the synthesis of $N$-hydroxyalkyl-3-acetoxy-2-phenyl indoles. $N$ Alkylation of 2-phenyl and 2-(p-fluoro)-phenyl indole 4 with TBS-protected bromo-alcohols proceeded in modest yield using $\mathrm{NaH}$ in DMF, followed by acetoxylation with LTA and removal of the TBS protecting group to give alcohols 9 (Scheme 4). The modest yield for the two steps was due to the low conversion of starting material in the acetoxylation reaction (see experimental).

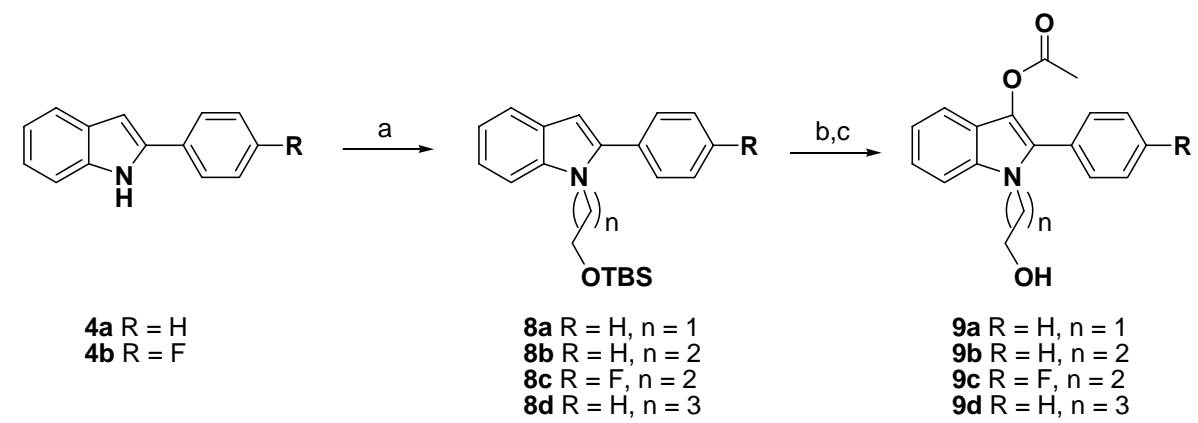

Scheme 4. Synthesis of cyclization precursors. a) $\mathrm{NaH}$, DMF, TBSO- $\left(\mathrm{CH}_{2}\right)_{\mathrm{n}}-\mathrm{Br}, \mathrm{n}=1,2,3$. b) $\mathrm{CH}_{2} \mathrm{Cl}_{2}, \mathrm{~Pb}(\mathrm{OAc})_{4}$. c) THF, TBAF.

Acidic hydrolysis of $N$-hydroxyalkyl-3-acetoxy indole derivatives 9 provided cyclic 2phenylindoxyl structures $\mathbf{1 0}$ and lent preliminary evidence to our hypothesis regarding the mechanism of the reaction (Scheme 5). All product structures were confirmed by a combination of $1 \mathrm{D}\left(\mathrm{DEPT},{ }^{13} \mathrm{C}\right)$ and 2D-NMR experiments (gCOSY, gHMBC, gHMQC). The formation of indoxyls 10a and 10d was also accompanied by the formation of a side product ${ }^{15}$ that accounted for the balance of the total yield in the reaction. The mechanism to account for the formation of the side product is currently under investigation and will be reported in due course. 


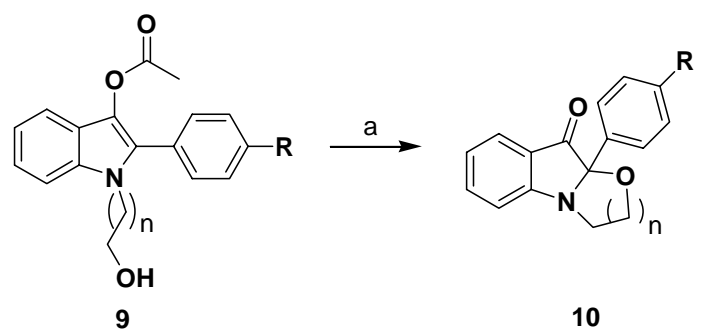

\begin{tabular}{ccccc}
\hline $\mathrm{n}$ & $\mathrm{R}$ & $\begin{array}{c}\text { Starting } \\
\text { material }\end{array}$ & $\begin{array}{c}\text { Cyclized } \\
\text { product }\end{array}$ & Yield \\
\hline 1 & $\mathrm{H}$ & $\mathbf{9 a}$ & $\mathbf{1 0 a}$ & $10 \%$ \\
2 & $\mathrm{H}$ & $\mathbf{9 b}$ & $\mathbf{1 0 b}$ & $76 \%$ \\
2 & $\mathrm{~F}$ & $\mathbf{9 c}$ & $\mathbf{1 0 c}$ & $55 \%$ \\
3 & $\mathrm{H}$ & $\mathbf{9 d}$ & $\mathbf{1 0 d}$ & $16 \%$ \\
\hline
\end{tabular}

Scheme 5. Cyclization reaction. a) $2 \mathrm{M} \mathrm{HCl}, \mathrm{MeOH}, \mathrm{RT} \rightarrow 70{ }^{\circ} \mathrm{C}$

Indoxyl 10b was also elaborated to show that it represented a potentially valuable scaffold for library synthesis. Reaction with MeLi and n-pentyl Grignard gave tertiary alcohols 11a and 11b by HPLC and GC-MS analysis (Scheme 6).

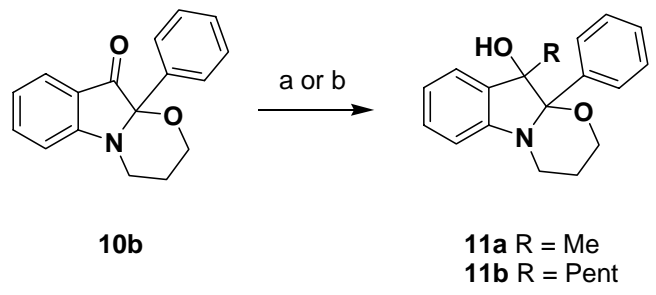

Scheme 6. Reaction with nucleophiles. a) MeMgBr, THF. b) Pent-MgBr, THF

In summary, we have detailed a new synthesis of 2-phenylindoxyls. Our initial work showed that 2,2'-substitution was the only possibility due to the ease with which 2phenylindoxyls were oxidized at the 2-position. $N$-hydroxyalkyl substitution provided novel cyclic 2-phenylindoxyl skeletons that could be elaborated with various nucleophiles. Work is underway to elucidate the mechanism under acidic conditions and will be reported in due course.

\section{Experimental Section}

General Procedures. All commercial reagents were used without further purification, unless otherwise noted. Anhydrous reactions were performed in flame-dried glassware under $\mathrm{N}_{2}$. NMR spectra were recorded on a Varian $400 \mathrm{MHz}$ spectrometer in deuterochloroform with 
trimethylsilane (TMS) as an internal reference. Silica gel column chromatography was performed using an ISCO Combiflash system with detection at $254 \mathrm{~nm}$ on ISCO normal phase silica gel cartridges. HPLC was performed on a Hewlett Packard Series 1100 pump connected to an Agilent Zorbax RX-C18 $5 \mu \mathrm{m}, 4.6$ X $250 \mathrm{~mm}$ column, with detection on a Hewlett Packard Series $1100 \mathrm{UV} / \mathrm{Vis}$ detector monitoring at 214 and $254 \mathrm{~nm}$. The following linear gradient was used for all samples: Flow rate $=1 \mathrm{ml} / \mathrm{min}$, Solvent $\mathrm{A}=\mathrm{H}_{2} 0 \mathrm{w} / 0.05 \% \mathrm{TFA}$, Solvent $\mathrm{B}=\mathrm{MeCN}$ $\mathrm{w} / 0.05 \%$ TFA. Time $0 \mathrm{~min}=5 \%$ Solvent B, time $4 \mathrm{~min}=40 \%$ Solvent B, time $8 \mathrm{~min}=100 \%$ Solvent B, $12 \mathrm{~min}=5 \%$ Solvent B, $20 \mathrm{~min}=5 \%$ Solvent B. GC-MS was performed on a Hewlett Packard 6890 Series GC System with an HP1 column (30 meters, $0.15 \mu$ film thickness) coupled to a Hewlett Packard 5973 Series Mass Selective Detector. The following linear temperature gradient was used: $100^{\circ} \mathrm{C}$ for 5 minutes, then $20^{\circ} \mathrm{C} / \mathrm{min}$ to $320^{\circ} \mathrm{C}$. Hold $@ 320^{\circ} \mathrm{C}$ for 10 minutes.

1-(Cyclopropylmethyl)-2-phenyl-1H-indol-3-yl acetate (5a). 2-Phenylindole (1.0 g, 5.17 mmol) was dissolved in anhydrous DMF $(50 \mathrm{~mL})$ and cooled to $0{ }^{\circ} \mathrm{C}$. $\mathrm{NaH}(60 \%$ dispersion in mineral oil, $248 \mathrm{mg}, 6.21 \mathrm{mmol}$ ) was added and the resulting yellow solution was stirred at $0^{\circ} \mathrm{C}$ for 20 minutes. Cyclopropylmethyl bromide $(600 \mu \mathrm{L}, 6.21 \mathrm{mmol})$ was added and the yellow solution was allowed to warm to RT over $16 \mathrm{~h}$. MeOH $(5 \mathrm{~mL})$ was added and the solution was poured into $\mathrm{H}_{2} \mathrm{O}(100 \mathrm{~mL})$ and washed with $\mathrm{Et}_{2} \mathrm{O}(3 \mathrm{X} 50 \mathrm{~mL})$. The combined organic extracts were washed with brine $(50 \mathrm{~mL})$, dried $\left(\mathrm{Na}_{2} \mathrm{SO}_{4}\right)$, filtered and concentrated. Purification by silica gel column chromatography with $0 \rightarrow 20 \%$ EtOAc/hexanes yielded 1-(cyclopropylmethyl)-2phenyl-1H-indole (292 mg, $23 \%$ ) as a clear oil. HPLC $\mathrm{R}_{\mathrm{t}}=11.44 \mathrm{~min},{ }^{1} \mathrm{H}$ NMR (400 MHz, $\left.\mathrm{CDCl}_{3}\right) 7.82(\mathrm{~d}, \mathrm{~J}=7.70 \mathrm{~Hz}, 1 \mathrm{H}), 7.67-7.54(\mathrm{~m}, 7 \mathrm{H}), 7.41$ (at, 1H), 7.32 (at, 1H), 6.72 (s, 1H), $4.20(\mathrm{~d}, \mathrm{~J}=6.23 \mathrm{~Hz}, 1 \mathrm{H}), 1.23-1.19(\mathrm{~m}, 1 \mathrm{H}), 0.54-0.49(\mathrm{~m}, 2 \mathrm{H}), 0.20-0.16(\mathrm{~m}, 2 \mathrm{H}) ;{ }^{13} \mathrm{C}$ NMR $\left(100 \mathrm{MHz}, \mathrm{CDCl}_{3}\right)$ 141.4, 137.8, 133.6, 129.8, 129.6, 128.6, 128.5, 128.4, 128.1, 121.7, 120.7, 120.0, 110.4, 102.6, 48.2, 11.6, 4.2; GC-MS $13.38 \mathrm{~min}, \mathrm{M}^{+}$247. 1-(cyclopropylmethyl)-2phenyl-1H-indole $(273 \mathrm{mg}, 1.11 \mathrm{mmol})$ was dissolved in anhydrous $\mathrm{CH}_{2} \mathrm{Cl}_{2}(11 \mathrm{~mL})$ and $\mathrm{Pb}(\mathrm{IV})$ acetate (539 mg, $1.22 \mathrm{mmol}$ ) was added. The brown solution was stirred at RT for $16 \mathrm{~h}$, then diluted with $\mathrm{CH}_{2} \mathrm{Cl}_{2}(30 \mathrm{~mL})$, washed with brine $(2 \mathrm{X} 20 \mathrm{~mL})$, dried $\left(\mathrm{Na}_{2} \mathrm{SO}_{4}\right)$, filtered and concentrated. Purification by silica gel column chromatography with $0 \rightarrow 10 \%$ EtOAc/hexanes yielded 5a (100 mg, $29 \%)$ as a white foam. HPLC $\mathrm{R}_{\mathrm{t}}=10.82 \mathrm{~min},{ }^{1} \mathrm{H} \mathrm{NMR}\left(400 \mathrm{MHz}, \mathrm{CDCl}_{3}\right)$ 7.52-7.44 (m, 7H), 7.29-7.25 (m, 1H), 7.17 (at, 1H), 3.99 (d, J = 6.60 Hz, 2H), 2.25 (s, 3H), 1.07$1.03(\mathrm{~m}, 1 \mathrm{H}), 0.42-0.37(\mathrm{~m}, 2 \mathrm{H}), 0.04-0.012(\mathrm{~m}, 2 \mathrm{H}) ;{ }^{13} \mathrm{C} \mathrm{NMR}\left(100 \mathrm{MHz}, \mathrm{CDCl}_{3}\right)$ 170.1, 134.6, $130.5,130.3$, 130.2 , 128.8, 128.7, 128.6, 122.6, 121.1, 120.2, 117.7, 110.7, 48.4, 20.8, 11.6, 4.4; GC-MS $14.3 \mathrm{~min}, \mathrm{M}^{+} 305$.

2-Phenyl-1-propyl-1H-indol-3-yl acetate (5b). 2-Phenyl-1-propyl-1H-indole (1.4 g, 5.95 mmol) was dissolved in anhydrous $\mathrm{CH}_{2} \mathrm{Cl}_{2}(60 \mathrm{~mL})$ and $\mathrm{Pb}(\mathrm{IV})$ acetate $(2.9 \mathrm{~g}, 6.6 \mathrm{mmol})$ was added. The brown solution was stirred at RT for $16 \mathrm{~h}$, then diluted with $\mathrm{CH}_{2} \mathrm{Cl}_{2}(100 \mathrm{~mL})$, washed with brine $(2 \mathrm{X} 100 \mathrm{~mL})$, dried $\left(\mathrm{Na}_{2} \mathrm{SO}_{4}\right)$, filtered and concentrated. Purification by silica gel column chromatography with $0 \rightarrow 5 \%$ EtOAc/hexanes yielded $\mathbf{5 b}$ (400 $\mathrm{mg}, 34 \%$ ) as a yellow solid. HPLC R $\mathrm{t}_{\mathrm{t}}=10.9 \mathrm{~min},{ }^{1} \mathrm{H}$ NMR $\left(400 \mathrm{MHz}, \mathrm{CDCl}_{3}\right)$ 7.54-7.41 (m, 7H), 7.28 (td, J = 6.97, 
$1.1 \mathrm{~Hz}, 1 \mathrm{H}), 7.21-7.17(\mathrm{~m}, 1 \mathrm{H}), 4.08$ (at, $2 \mathrm{H}), 2.27(\mathrm{~s}, 3 \mathrm{H}), 1.76-1.70(\mathrm{~m}, 2 \mathrm{H}), 0.80(\mathrm{at}, 3 \mathrm{H}) ;{ }^{13} \mathrm{C}$ NMR (100 MHz, $\left.\mathrm{CDCl}_{3}\right)$ 170.0, 134.3, 130.2, 130.0, 128.8, 128.5, 126.8, 122.4, 121.0, 120.1, 117.7, 110.4, 45.7, 23.5, 20.7, 11.4; GC-MS 13.7 min, $\mathrm{M}^{+} 293$.

2-Methoxy-2-phenyl-1-propylindolin-3-one (6b) and 2-hydroxy-2-phenyl-1-propylindolin3-one (7b). 5b (155 mg, $0.53 \mathrm{mmol})$ was suspended in $\mathrm{MeOH}(10 \mathrm{~mL})$ and $2.0 \mathrm{M} \mathrm{HCl}(530 \mu \mathrm{L}$, $1.06 \mathrm{mmol}$ ) was added. The solution was shaken at RT for 72 hours, then concentrated and purified by silica gel column chromatography with $0 \rightarrow 20 \%$ EtOAc/hexanes to give $6 \mathbf{b}$ (73 mg) as an orange oil and $7 \mathbf{b}(21 \mathrm{mg})$ as an orange oil. $\mathbf{6 b}$ : HPLC $\mathrm{R}_{\mathrm{t}}=10.82 \mathrm{~min},{ }^{1} \mathrm{H}$ NMR $(400 \mathrm{MHz}$, $\left.\mathrm{CDCl}_{3}\right) 7.56-7.30(\mathrm{~m}, 7 \mathrm{H}), 6.82(\mathrm{~d}, \mathrm{~J}=8.43 \mathrm{~Hz}, 1 \mathrm{H}), 6.74(\mathrm{t}, \mathrm{J}=7.33 \mathrm{~Hz}, 1 \mathrm{H}), 3.31(\mathrm{~s}, 3 \mathrm{H})$, 3.25-3.17 (m, 1H), 3.09-3.03 (m, 1H), 1.65-1.59 (m, 2H), $0.89(\mathrm{t}, \mathrm{J}=7.33 \mathrm{~Hz}, 3 \mathrm{H}) ;{ }^{13} \mathrm{C}$ NMR $\left(100 \mathrm{MHz}, \mathrm{CDCl}_{3}\right)$ 199.0, 161.7, 138.8, 136.1, 128.8, 128.7, 126.4, 125.5, 118.5, 117.8, 107.9, 96.2, 51.7, 44.9, 22.2, 11.8; GC-MS $12.21 \mathrm{~min}, \mathrm{M}^{+}$281. 7b: HPLC R $\mathrm{t}_{\mathrm{t}}=9.86 \mathrm{~min},{ }^{1} \mathrm{H} \mathrm{NMR}(400$ $\left.\mathrm{MHz}, \mathrm{CDCl}_{3}\right)$ 7.53-7.49 (m, 2H), 7.41-7.32 (m, 4H), 6.77-6.71 (m, 2H), 3.32-3.25 (m, 1H), 3.10$3.02(\mathrm{~m}, 1 \mathrm{H}), 1.63-1.57(\mathrm{~m}, 2 \mathrm{H}), 0.86(\mathrm{at}, 3 \mathrm{H}) ;{ }^{13} \mathrm{C} \mathrm{NMR}\left(100 \mathrm{MHz}, \mathrm{CDCl}_{3}\right)$ 200.3, 160.9, $138.8,136.5,128.8,128.7,126.1,126.1,117.8,117.0,108.1,91.3,44.6,22.3,11.6$; GC-MS 13.3 $\min , \mathrm{M}^{+} 267$.

1-(Cyclopropylmethyl)-2-methoxy-2-phenylindolin-3-one (6a) and 1-(Cyclopropylmethyl)2-hydroxy-2-phenylindolin-3-one (7a). 6a: HPLC R $\mathrm{R}_{\mathrm{t}}=10.8 \mathrm{~min}, \mathrm{GC}-\mathrm{MS} 13.88 \mathrm{~min}, \mathrm{M}^{+} 293$. 7a: $\mathrm{HPLC} \mathrm{R}_{\mathrm{t}}=9.91 \mathrm{~min}, \mathrm{GC}-\mathrm{MS} 13.93 \mathrm{~min}, \mathrm{M}^{+} 279$.

Representative procedures for sequence of $\mathbf{4} \rightarrow \mathbf{8}$ (alkylation) and $\mathbf{8} \rightarrow \mathbf{9}$ (acetoxylation)

1-(2-tert-Butyldimethylsilyloxyethyl)-2-phenyl-1H-indole (8a). 2-Phenylindole (4a) (2.2 g, $11.7 \mathrm{mmol})$ was dissolved in anhydrous DMF $(50 \mathrm{~mL})$ and cooled to $0^{\circ} \mathrm{C}$. $\mathrm{NaH}(60 \%$ dispersion in mineral oil, $702 \mathrm{mg}, 17.5 \mathrm{mmol}$ ) was added and the resulting yellow solution was stirred at 0 ${ }^{\circ} \mathrm{C}$ for 20 minutes. 2-tert-butyldimethylsilyl-1-bromoethanol (2.72 g, $\left.11.4 \mathrm{mmol}\right)$ was dissolved in DMF $(10 \mathrm{~mL})$ and added dropwise to the indole solution. The yellow solution was allowed to warm to RT over $16 \mathrm{~h}$. MeOH (5 mL) was added and the solution was poured into $\mathrm{H}_{2} \mathrm{O}(100 \mathrm{~mL})$ and washed with $\mathrm{Et}_{2} \mathrm{O}(3 \mathrm{X} 50 \mathrm{~mL})$. The combined organic extracts were washed with brine (50 $\mathrm{mL})$, dried $\left(\mathrm{Na}_{2} \mathrm{SO}_{4}\right)$, filtered and concentrated. Purification by silica gel column chromatography with $0 \rightarrow 5 \%$ EtOAc/hexanes yielded 8a $(1.69 \mathrm{~g}, 41 \%)$ as a clear oil. HPLC $\mathrm{R}_{\mathrm{t}}=$ $13.3 \mathrm{~min},{ }^{1} \mathrm{H}$ NMR (400 MHz, $\left.\mathrm{CDCl}_{3}\right) 7.65$ (d, J = 7.70 Hz, 1H), 7.59-7.57 (m, 2H), 7.51-7.43 (m, 4H), 7.27-7.23 (m, 1H), $7.17($ at, $1 \mathrm{H}), 6.56(\mathrm{~d}, \mathrm{~J}=1.47 \mathrm{~Hz}, 1 \mathrm{H}), 4.31(\mathrm{td}, \mathrm{J}=1.83,6.60 \mathrm{~Hz}$, $2 \mathrm{H}), 3.86-3.82(\mathrm{~m}, 2 \mathrm{H}), 0.80(\mathrm{~s}, 9 \mathrm{H}),-0.13(\mathrm{~s}, 6 \mathrm{H}) ;{ }^{13} \mathrm{C} \mathrm{NMR}\left(100 \mathrm{MHz}, \mathrm{CDCl}_{3}\right)$ 141.6, 138.0, $133.2,129.9,128.7,128.3,128.2,121.8,120.7,120.1,110.4,102.6,62.0,46.1,26.0$, -5.5; GCMS $14.1 \mathrm{~min}, \mathrm{M}^{+} 351$.

1-(2-Hydroxyethyl)-2-phenyl-1H-indol-3-yl acetate (9a). 8a (1.69 g, 4.8 mmol) was dissolved in anhydrous $\mathrm{CH}_{2} \mathrm{Cl}_{2}(50 \mathrm{~mL})$ and $\mathrm{Pb}$ (IV) acetate $(2.35 \mathrm{~g}, 5.3 \mathrm{mmol})$ was added. The brown solution was stirred at RT for $16 \mathrm{~h}$, then diluted with $\mathrm{CH}_{2} \mathrm{Cl}_{2}(100 \mathrm{~mL})$, washed with brine $(2 \mathrm{X}$ $100 \mathrm{~mL})$, dried $\left(\mathrm{Na}_{2} \mathrm{SO}_{4}\right)$, filtered and concentrated. Purification by silica gel column chromatography with $0 \rightarrow 5 \%$ EtOAc/hexanes yielded 1-(2-tert-butyldimethylsilyloxyethyl)-2phenyl-1H-indol-3-yl acetate (560 mg, $28 \%$ ) as a yellow solid. HPLC $\mathrm{R}_{\mathrm{t}}=12.1 \mathrm{~min},{ }^{1} \mathrm{H}$ NMR 
$\left(400 \mathrm{MHz}, \mathrm{CDCl}_{3}\right)$ 7.57-7.47 (m, 7H), 7.30 (at, 1H), 7.20 (at, 1H), $4.27(\mathrm{t}, \mathrm{J}=6.60 \mathrm{~Hz}, 2 \mathrm{H}), 3.83$ (at, 2H), $2.29(\mathrm{~s}, 3 \mathrm{H}), 0.84(\mathrm{~s}, 9 \mathrm{H}),-0.08(\mathrm{~s}, 6 \mathrm{H}) ;{ }^{13} \mathrm{C} \mathrm{NMR}\left(100 \mathrm{MHz}, \mathrm{CDCl}_{3}\right)$ 169.9, 134.8, 130.3, 129.7, 128.8, 128.6, 127.1, 122.5, 121.0, 120.2, 117.6, 110.6, 62.0, 46.0, 25.9, 20.7, -5.54; GC-MS $14.75 \mathrm{~min}, \mathrm{M}^{+}$409. 1-(2-tert-butyldimethylsilyloxyethyl)-2-phenyl-1H-indol-3-yl acetate $(558 \mathrm{mg}, 1.36 \mathrm{mmol})$ was dissolved in anhydrous THF $(14 \mathrm{~mL})$ and $1.0 \mathrm{M}$ TBAF in THF ( $2 \mathrm{~mL}, 2.0 \mathrm{mmol}$ ) was added. The clear orange solution was stirred for $40 \mathrm{~min}$ at RT, diluted with EtOAc and washed with $\mathrm{H}_{2} \mathrm{O}(2 \mathrm{X} 50 \mathrm{~mL})$ and brine $(50 \mathrm{~mL})$. The organic extracts were dried $\left(\mathrm{Na}_{2} \mathrm{SO}_{4}\right)$, filtered and concentrated. Purification by silica gel column chromatography with $0 \rightarrow 40 \%$ EtOAc/hexanes yielded 9a (142 mg, 35\%) as a yellow oil. HPLC $\mathrm{R}_{\mathrm{t}}=9.42 \mathrm{~min},{ }^{1} \mathrm{H}$ NMR (400 MHz, $\mathrm{CDCl}_{3}$ ) 7.43-7.33 (m, 6H), 7.20 (at, 1H), 7.11 (at, 1H), 4.03 (t, J = 5.87 Hz, 2H), 3.53 (at, 2H), 2.20 (s, 3H); ${ }^{13} \mathrm{C}$ NMR (100 MHz, $\left.\mathrm{CDCl}_{3}\right)$ 170.7, 134.5, 130.3, 130.3, 129.4, $128.8,128.7,126.8,122.7,120.9,120.4,117.5,110.6,61.2,45.8,20.7$; GC-MS 13.9 min, $\mathrm{M}^{+}$ 295.

1-(3-tert-Butyldimethylsilyloxypropyl)-2-phenyl-1H-indole (8b). This compound was obtained as a clear oil in 53\% yield using the general alkylation procedure outlined above. 8b: HPLC R $\mathrm{t}_{\mathrm{t}}=14.02 \mathrm{~min},{ }^{1} \mathrm{H}$ NMR $\left(400 \mathrm{MHz}, \mathrm{CDCl}_{3}\right) 7.72(\mathrm{~d}, \mathrm{~J}=8.07 \mathrm{~Hz}, 1 \mathrm{H}), 7.59-7.46(\mathrm{~m}$, $5 \mathrm{H}), 7.30$ (at, 1H), $7.21(\mathrm{t}, \mathrm{J}=7.70 \mathrm{~Hz}, 1 \mathrm{H}), 6.61(\mathrm{~s}, 1 \mathrm{H}), 4.36(\mathrm{t}, \mathrm{J}=7.70 \mathrm{~Hz}, 2 \mathrm{H}), 3.60(\mathrm{t}, \mathrm{J}=$ $5.87 \mathrm{~Hz}, 2 \mathrm{H}), 1.97-1.92(\mathrm{~m}, 2 \mathrm{H}), 0.95(\mathrm{~s}, 3 \mathrm{H}), 0.067(\mathrm{~s}, 5 \mathrm{H}) ;{ }^{13} \mathrm{C}$ NMR $\left(100 \mathrm{MHz}, \mathrm{CDCl}_{3}\right)$ $141.4,137.7,133.4,129.5,128.6,128.3,128.1,121.7,120.7,120.0,110.3,102.4,60.4,41.3$, 33.3, 26.1, -5.25; GC-MS $11.8 \mathrm{~min}, \mathrm{M}^{+} 365$.

1-(3-Hydroxypropyl)-2-phenyl-1H-indol-3-yl acetate (9b). This compound was obtained as a colorless solid in $25 \%$ yield using the general acetoxylation procedure outlined above. 9b: HPLC $\mathrm{R}_{\mathrm{t}}=9.46 \mathrm{~min},{ }^{1} \mathrm{H} \mathrm{NMR}\left(400 \mathrm{MHz}, \mathrm{CDCl}_{3}\right)$ 7.51-7.42 (m, 7H), 7.26-7.13 (m, 3H), 4.28-4.25 (m, 2H), $3.42(\mathrm{~s}, 3 \mathrm{H}), 2.24(\mathrm{~s}, 3 \mathrm{H}), 1.86-1.82(\mathrm{~m}, 2 \mathrm{H}) ;{ }^{13} \mathrm{C}$ NMR (100 MHz, $\left.\mathrm{CDCl}_{3}\right)$ 170.1, 134.4, $130.1,129.9,129.8,128.9,128.7,127.0,122.6,121.0,120.2,117.7,110.4,59.6,40.5,32.7$, 20.7; GC-MS 11.6 min, $\mathrm{M}^{+} 309$.

1-(3-tert-Butyldimethylsilyloxypropyl)-2-(4-fluorophenyl)-1H-indole (8c). This compound was obtained as a clear oil in $16 \%$ yield using the general alkylation procedure outlined above. 8c: HPLC $\mathrm{R}_{\mathrm{t}}=13.68 \mathrm{~min},{ }^{1} \mathrm{H}$ NMR $\left(400 \mathrm{MHz}, \mathrm{CDCl}_{3}\right) 7.64(\mathrm{~d}, \mathrm{~J}=7.70 \mathrm{~Hz}, 1 \mathrm{H}), 7.48-7.43$ (m, 4H), 7.23 (at, 1H), 7.17-7.12 (m, 3H), 6.50 (s, 1H), 4.25 (t, J = 7.70 Hz, 2H), 3.53 (at, 2H), 1.90$1.84(\mathrm{~m}, 2 \mathrm{H}), 0.87(\mathrm{~s}, 10 \mathrm{H}), 0.00(\mathrm{~s}, 6 \mathrm{H}) ;{ }^{13} \mathrm{C} \mathrm{NMR}\left(100 \mathrm{MHz}, \mathrm{CDCl}_{3}\right)$ 164.0, 161.6, 140.3, 137.6, 131.3, 131.3, 129.5, 121.8, 120.7, 120.1, 115.8, 115.6, 110.3, 102.5, 60.3, 41.3, 33.3, 26.0, -5.3; GC-MS $11.8 \mathrm{~min}, \mathrm{M}^{+} 383$.

2-(4-Fluorophenyl)-1-(3-hydroxypropyl)-1H-indol-3-yl acetate (9c). This compound was obtained as a yellowish solid in $61 \%$ yield using the general acetoxylation procedure outlined above. HPLC $\mathrm{R}_{\mathrm{t}}=9.54 \mathrm{~min},{ }^{1} \mathrm{H}$ NMR (400 MHz, $\left.\mathrm{CDCl}_{3}\right)$ 7.41-7.38 (m, 4H), 7.25-7.14 (m, 4H), $4.17(\mathrm{t}, \mathrm{J}=6.97 \mathrm{~Hz}, 1 \mathrm{H}), 3.38(\mathrm{t}, \mathrm{J}=5.87 \mathrm{~Hz}, 1 \mathrm{H}), 2.23(\mathrm{~s}, 3 \mathrm{H}), 1.79-1.76(\mathrm{~m}, 2 \mathrm{H}), 1.40$ (bs, $1 \mathrm{H}) ;{ }^{13} \mathrm{C}$ NMR $\left(100 \mathrm{MHz}, \mathrm{CDCl}_{3}\right)$ 170.0, 164.2, 161.7, 134.3, 132.0, 131.9, 128.9, 125.8, 125.8, $122.8,120.9,120.3,117.7,116.2,116.0,110.4,59.7,40.6,32.7,20.7$; GC-MS 14.7 min, $\mathrm{M}^{+}$ 327. 
1-(4-Hydroxybutyl)-2-phenyl-1H-indol-3-yl acetate (9d). This compound was obtained as a clear oil in 48\% yield using the general acetoxylation procedure outlined above. 9d: HPLC $\mathrm{R}_{\mathrm{t}}=$ $9.55 \mathrm{~min},{ }^{1} \mathrm{H}$ NMR (400 MHz, $\left.\mathrm{CDCl}_{3}\right)$ 7.51-7.38 (m, 7H), 7.25 (at, 1H), 7.16 (at, 1H), 4.12 (t, J $=7.33 \mathrm{~Hz}, 2 \mathrm{H}), 3.38(\mathrm{t}, \mathrm{J}=6.23 \mathrm{~Hz}, 2 \mathrm{H}), 2.25(\mathrm{~s}, 3 \mathrm{H}), 1.73-1.66(\mathrm{~m}, 2 \mathrm{H}), 1.34-1.30(\mathrm{~m}, 2 \mathrm{H}) ;{ }^{13} \mathrm{C}$ NMR (100 MHz, $\left.\mathrm{CDCl}_{3}\right)$ 170.1, 134.2, 130.1, 130.0, 129.9, 128.8, 128.6, 126.8, 122.5, 121.0, $120.1,117.7,62.1,43.7,29.7,26.4,20.7$; GC-MS $12.4 \mathrm{~min}, \mathrm{M}^{+} 323$.

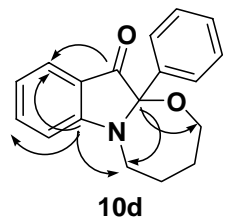

Representative procedure ("cyclization") for synthesis of compounds 10a-d: 9d (74 mg, 0.23 mmol) was suspended in $\mathrm{MeOH}(2 \mathrm{~mL})$ and $2.0 \mathrm{M} \mathrm{HCl}(230 \mu \mathrm{L}, 0.46 \mathrm{mmol})$ was added. The solution was shaken at RT for 16 hours, then $6 \mathrm{M} \mathrm{HCl}(12$ drops, ca. $60 \mu \mathrm{L})$ was added and the temperature was increased to $70^{\circ} \mathrm{C}$. After $5.5 \mathrm{~h}$ the solution was cooled to RT and poured into sat'd aqueous $\mathrm{NaHCO}_{3}(15 \mathrm{~mL})$ and washed with $\mathrm{Et}_{2} \mathrm{O}(3 \mathrm{X} 30 \mathrm{~mL})$. The combined organic washes were washed with brine $(20 \mathrm{~mL})$, dried $\left(\mathrm{Na}_{2} \mathrm{SO}_{4}\right)$, filtered and concentrated. Purification by silica gel column chromatography with $0 \rightarrow 25 \%$ EtOAc/hexanes yielded 10d (10 mg, 16\%) as a fluorescent green oil. 10d: HPLC $\mathrm{R}_{\mathrm{t}}=10.32 \mathrm{~min},{ }^{1} \mathrm{H} \mathrm{NMR}\left(400 \mathrm{MHz}, \mathrm{CDCl}_{3}\right)$ 7.57-7.50 (m, 4H), 7.36-7.31 (m, 3H), $6.84(\mathrm{~d}, J=8.06 \mathrm{~Hz}, 1 \mathrm{H}), 6.74(\mathrm{t}, J=7.33 \mathrm{~Hz}, 1 \mathrm{H}), 3.98-3.93$ (m, $1 \mathrm{H}), 3.89-3.85(\mathrm{~m}, 1 \mathrm{H}), 3.24-3.18(\mathrm{~m}, 2 \mathrm{H}), 1.83-1.67(\mathrm{~m}, 4 \mathrm{H}) ;{ }^{13} \mathrm{C}$ NMR $\left(100 \mathrm{MHz}, \mathrm{CDCl}_{3}\right)$ 200.9, 160.7, 138.5, 136.7, 129.0, 128.8, 126.5, 125.8, 119.2, 118.2, 108.9, 94.9, 66.3, 41.4, 30.5, 24.7; GC-MS $11.5 \mathrm{~min}, \mathrm{M}^{+} 279$. Selected gHMBC couplings (see above): $200.9 \rightarrow 7.5 ; 160.7 \rightarrow$ $3.2,7.4,7.5 ; 94.9 \rightarrow 3.2,3.8,3.9$.

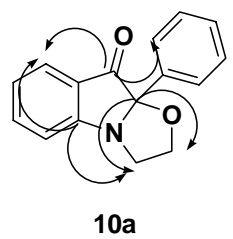

This compound was obtained as a fluorescent green oil in $10 \%$ yield using the general cyclization procedure outlined above. 10a: $\mathrm{HPLC} \mathrm{R}_{\mathrm{t}}=9.87 \mathrm{~min},{ }^{1} \mathrm{H}$ NMR $\left(400 \mathrm{MHz}, \mathrm{CDCl}_{3}\right)$ 7.66-7.60 (m, 4H), 7.41-7.37 (m, 3H), 7.11-7.05 (m, 2H), 4.10-4.04 (m, 2H), 3.69-3.63 (m, 1H), 3.58-3.51 (m, 1H); ${ }^{13} \mathrm{C}$ NMR (100 MHz, $\left.\mathrm{CDCl}_{3}\right)$ 197.2, 163.9, 137.8, 136.0, 128.9, 128.4, 126.5, 125.7, 122.8, 122.6, 114.8, 99.6, 67.9, 50.1; GC-MS 13.4 min, $\mathrm{M}^{+}$251. Selected gHMBC couplings (see above): $200 \rightarrow 7.62,7.64 ; 164 \rightarrow 3.65,3.5,7.64 ; 99 \rightarrow 3.65,4.05$. 


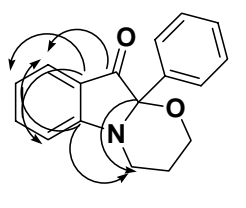

10b

This compound was obtained as a yellow solid in $76 \%$ yield using the general cyclization procedure outlined above. 10b: HPLC $\mathrm{R}_{\mathrm{t}}=10.0 \mathrm{~min},{ }^{1} \mathrm{H} \mathrm{NMR}\left(400 \mathrm{MHz}, \mathrm{CDCl}_{3}\right)$ 7.60-7.51 (m, $4 \mathrm{H}), 7.44-7.36(\mathrm{~m}, 3 \mathrm{H}), 6.91(\mathrm{~d}, \mathrm{~J}=8.43 \mathrm{~Hz}, 1 \mathrm{H}), 6.80(\mathrm{at}, 1 \mathrm{H}), 3.96-3.81(\mathrm{~m}, 3 \mathrm{H}), 3.46-3.39$ (m, 1H), 2.05-1.97 (m, 1H), $1.40(\mathrm{dd}, \mathrm{J}=1.1,13.2 \mathrm{~Hz}, 1 \mathrm{H}) ;{ }^{13} \mathrm{C}$ NMR $\left(100 \mathrm{MHz}, \mathrm{CDCl}_{3}\right)$ 196.4, 161.0, 138.0, 133.9, 129.2, 127.8, 126.6, 119.0, 118.9, 109.4, 90.6, 63.0, 40.0, 24.7; GC-MS $14.04 \mathrm{~min}, \mathrm{M}^{+} 265$. Selected gHMBC couplings (see above): $197 \rightarrow 7.6 ; 160 \rightarrow 3.5,7.6 ; 119 \rightarrow$ $6.8,6.98 ; 90 \rightarrow 4.0$.

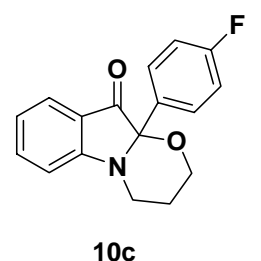

This compound was obtained as a fluorescent green oil in 55\% yield using the general cyclization procedure outlined above. 10c: HPLC $\mathrm{R}_{\mathrm{t}}=10.1 \mathrm{~min} ;{ }^{1} \mathrm{H}$ NMR $\left(400 \mathrm{MHz}, \mathrm{CDCl}_{3}\right)$ 7.61-7.52 (m, 4H), $7.10($ at, $2 \mathrm{H}), 6.92(\mathrm{~d}, \mathrm{~J}=8.43,1 \mathrm{H}), 6.83(\mathrm{at}, 1 \mathrm{H}), 3.97-3.90(\mathrm{~m}, 2 \mathrm{H}), 3.85-$ $3.78(\mathrm{~m}, 1 \mathrm{H}), 3.45-3.37(\mathrm{~m}, 1 \mathrm{H}), 2.05-1.95(\mathrm{~m}, 1 \mathrm{H}), 1.60-1.39(\mathrm{~m}, 1 \mathrm{H}) ;{ }^{13} \mathrm{C} \mathrm{NMR}(100 \mathrm{MHz}$, $\left.\mathrm{CDCl}_{3}\right)$ 196.2, 165.3, 162.0, 160.9, 138.0, 129.8, 129.7, 126.7, 119.3, 119.0, 116.3, 116.0, 109.6, 90.2, 62.9, 40.1, 24.6; GC-MS 12.35 min, $\mathrm{M}^{+} 283$.

\section{Acknowledgements}

The authors thank Scott Wilkinson for help with NMR experiments and interpretation of spectra and Drs. Scott Malcolm and FengJiang Wang for helpful discussions.

\section{References and Notes}

1. Horton, D. A.; Bourne, G. T.; Smythe, M. L. Chem Rev. 2003, 103, 893.

2. Sundberg, R. J. Indoles; Academic Press: San Diego, CA, 1996; Gribble, G. W. J. Chem. Soc. Perkin Trans. 1 2000, 1045.

3. For a recent example see Pearson, W. H.; Lee, I. Y.; Mi, Y.; Stoy, P. J. Org. Chem. 2004, 69, 9109. 
4. Alcaide, B.; Almendos, P.; Rodriguez-Acebes, R. J. Org. Chem. 2006, 71, 2346.

5. Witkop, B.; Ek, A. J. Am. Chem. Soc. 1951, 73, 5664.

6. Berti, C.; Greci, L.; Marchetti, L. J. Chem. Soc. Perkins Trans. 2 1979, 233.

7. Astolfi, P.; Bruni, P.; Greci, L.; Stipa, Pierluigi, S.; Righi, L.; Rizzoli, C. Eur. J. Org. Chem. 2003, 2626.

8. Merour, J. Y.; Chichereau, L.; Finet, J. P. Tetrahedron Lett. 1992, 33, 3867.

9. Bourlot, A. S.; Desarbre, E.; Merour, J. Y. Synthesis 1994, 411.

10. Pretka, J. E.; Lindwall, H. G. J. Org. Chem. 1954, 19, 1080.

11. Lednicer, D.; Emmert, D. E. J. Heterocyclic Chem 1970, 7, 575.

12. Sukari, M. A.; Vernon, J. A. J. Chem. Soc. Perkin Trans. 1 1983, 2219.

13. Russell, G. A.; Kaupp, G. J. Am. Chem. Soc. 1969, 91, 3851.

14. N-propyl dioxindole:

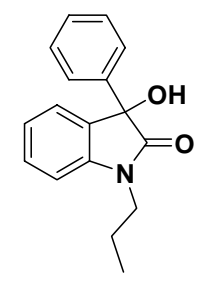

HPLC R $\mathrm{R}_{\mathrm{t}}=9.31 \mathrm{~min},{ }^{1} \mathrm{H} \mathrm{NMR}\left(400 \mathrm{MHz}, \mathrm{CDCl}_{3}\right)$ 7.39-7.26 (m, 6H), $7.06($ at, $1 \mathrm{H}), 6.91(\mathrm{~d}$, $J=7.70 \mathrm{~Hz}, 1 \mathrm{H}), 3.81-3.74(\mathrm{~m}, 1 \mathrm{H}), 3.67-3.61(\mathrm{~m}, 1 \mathrm{H}), 3.51(\mathrm{~s}, 1 \mathrm{H}), 1.79-1.70(\mathrm{~m}, 2 \mathrm{H})$, $0.98(\mathrm{t}, J=7.33 \mathrm{~Hz}, 3 \mathrm{H}) ;{ }^{13} \mathrm{C} \mathrm{NMR}\left(100 \mathrm{MHz}, \mathrm{CDCl}_{3}\right)$ 177.5, 143.0, 140.3, 131.8, 129.8, 128.6, 128.2, 125.2, 125.1, 123.3, 109.0, 77.9, 41.9, 20.7, 11.4; GC-MS 10.4 min, $\mathrm{M}^{+} 267$.

15. The structure of the side product has been tentatively identified:

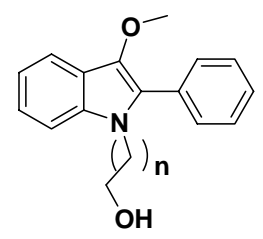

Analytical data for $\mathrm{n}=2$ : $\mathrm{HPLC} \mathrm{R}_{\mathrm{t}}=9.67 \mathrm{~min},{ }^{1} \mathrm{H}$ NMR $\left(400 \mathrm{MHz}, \mathrm{CDCl}_{3}\right) 7.69(\mathrm{~d}, J=$ $7.83 \mathrm{~Hz}, 1 \mathrm{H}), 7.64-7.37$ (m, 7H), 7.26-7.22 (m, 1H), 7.16-7.12 (at, 1H), 4.22 (at, 2H), 3.79 (s, 3H), 3.75 (at, 2H); ${ }^{13} \mathrm{C} \mathrm{NMR}\left(100 \mathrm{MHz}, \mathrm{CDCl}_{3}\right)$ 137.4, 134.6, 130.4, 128.6, 128.4, 128.1, 122.3, 121.1, 119.5, 118.1, 110.1, 62.0, 61.9, 45.8; GC-MS 14.1 min, $\mathrm{M}^{+} 267$. 